http://jmscr.igmpublication.org/home/

ISSN (e)-2347-176x ISSN (p) 2455-0450

crossref DOI: https://dx.doi.org/10.18535/jmscr/v10i1.34

Journal Of Medical Science And Clinical Research

\title{
Absolute Eosinophil Count versus C Reactive Protein as a Diagnostic and Prognostic Marker on Sepsis among in Patients of a Tertiary Care Centre in South Kerala
}

\author{
Authors \\ Dr Udayamma KP $\mathbf{P}^{\mathbf{1}}$, Dr Lalas $\mathrm{VS}^{2}$ \\ ${ }^{1}$ Additional professor of Medicine, Government TD Medical College, Alappuzha \\ ${ }^{2}$ Former resident, Department of Medicine, Government TD Medical College, Alappuzha \\ *Corresponding Author \\ Dr RNK Sankar \\ Assistant Professor, Department of Medicine, Government Medical College, Konni
}

\begin{abstract}
Introduction: Sepsis exists on a continuum of severity, ranging from infection and bacteremia to sepsis and septic shock, which can lead to multiple organ dysfunction syndrome (MODS) and death. The clinical and biologic phenotype of sepsis is modified by pre existing illness, co morbid conditions, medications and interventions. Overall mortality among patients admitted with sepsis in the ICU will be around $62 \%$. Since cultures are positive only in $<50 \%$ of the cases, various biochemical markers are evaluated to improve the diagnostic sensitivity and prognosis. This study compared the usefulness of Absolute Eosinophil Count (AEC) and C Reactive Protein (CRP) Materials and Methods: A prospective case control study was done using 61 cases and controls. The cut off for AEC was 35 and the cut off of CRP was 6. The cases were followed up till their end point in the hospital, defined as cured or died. The sensitivity, specificity and predictive values of AEC and CRP in diagnosing sepsis at the defined cut off was calculated using ROC curve. The prognostic accuracy was also calculated.

Observations: The mortality rate of sepsis in our institution is $83.6 \%$. The sensitivity, specificity, Positive and Negative Predictive Values of Absolute Eosinophil count in diagnosis of sepsis is 57.4\%, 98.4\%, 97.2\% and 69.8\% respectively.The sensitivity, specificity, Positive Predictive Value and Negative Predictive Value of C Reactive protein is $91.8 \%, 91.8 \%, 91.8 \%$ and $99.8 \%$ respectively.. Of the 51 deaths, 46 had CRP more than 6. Among the 10 cases cured, all had a CRP more than 6. Absolute Eosinophil Count of < 50 was seen in 29 of the 5eaths, and 6 of the 10 cases who were cured also had an AEC of < 50. So according to our study, both CRP and AEC are poor predictors of prognosis in sepsis.
\end{abstract}

\section{Introduction}

Sepsis, a syndrome of physiologic, pathologic and biochemical abnormalities induced by infection, is a major public health concern ${ }^{(1)}$. Sepsis exists on a continuum of severity, ranging from infection and bacteremia to sepsis and septic shock, which can lead to multiple organ dysfunction syndrome (MODS) and death. The clinical and biologic phenotype of sepsis is modified by pre existing illness, co morbid conditions, medications and interventions. $^{(1)}$

The causative organism for sepsis can be virus, bacteria, fungus or even parasite. Host factors like alcoholism, diabetes mellitus, chronic kidney disease, malignancies, immunosuppressive treatment and advanced age are risk factors for developing sepsis.

The definitions of sepsis have rapidly evolved since the early 1990s. The Systemic Inflammatory 
Response Syndrome is no longer included in the definition since it is not always caused by infection.

Overall mortality among patients admitted with sepsis in the ICU will be around $62 \%$. Because of the high mortality, early detection is of utmost importance. Since cultures are positive only in < $50 \%$ of the cases, various biochemical markers are evaluated to improve the diagnostic sensitivity and prognosis.

The present study therefore compares the utility of Absolute Eosinophil Count (AEC) Vs C reactive protein( CRP) in the diagnosis and prognosis of Sepsis.

\section{Aims and Objectives}

1. To compare Absolute Eosinophil Count and CRP as a diagnostic and prognostic marker in sepsis.

2. To assess the validity of Absolute Eosinophil Count in sepsis as a diagnostic and prognostic marker in sepsis.

\section{Materials and Methods}

It is a prospective observational case control study. The sample size calculated was 60 . The sample size decided was 65 .
Patients admitted in a Tertiary care centre in South Kerala with suspected sepsis who satisfied the inclusion criteria were studied.

Cases

\section{Inclusion Criteria}

1. Patients $>18$ years of age who satisfied the diagnostic criteria of sepsis by the qSOFA criteria.

\section{Exclusion Criteria}

1. Patients with HIV, TB and Malignancy.

2. Patients on long term immunosuppression.

3. Those not giving consent.

\section{Controls}

65 age and sex matched controls without any acute illness were taken as controls. They satisfied most of the exclusion criteria.

All the patients who satisfied the inclusion criteria were studied with a detailed clinical examination as well as CRP and Absolute Eosinophil Count. Of the 65 patients who got enrolled, 4 were taken to other centres and hence were lost for follow up. Hence the sample size was 61 in both cases and controls.

\section{Observations}

Figure 1: Percentage Distribution of cases according to Age

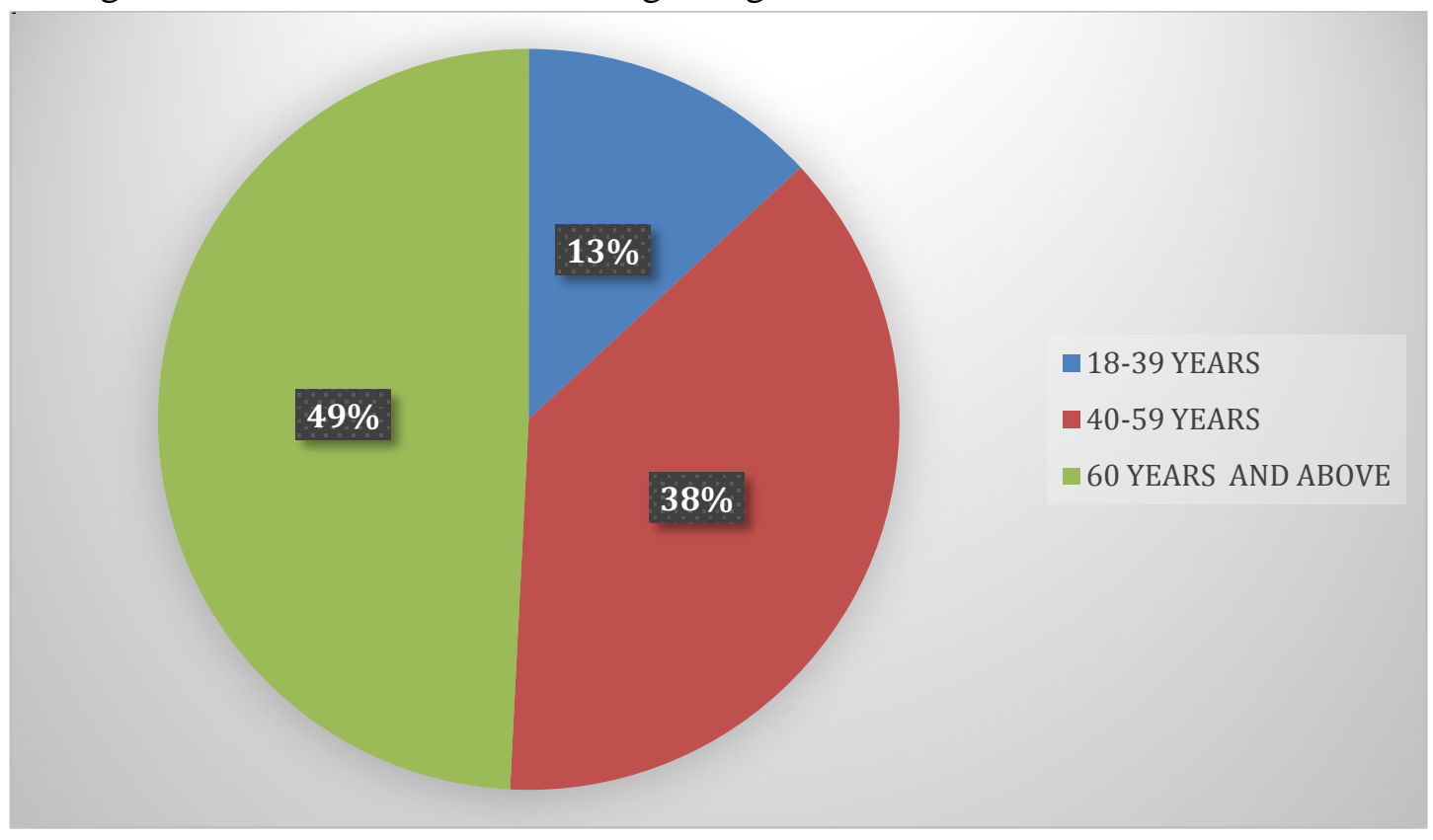




\section{JMSCR Vol||10||Issue||01||Page 213-219||January}

Figure 2: Percentage Distribution of Cases according to qSOFA Score:

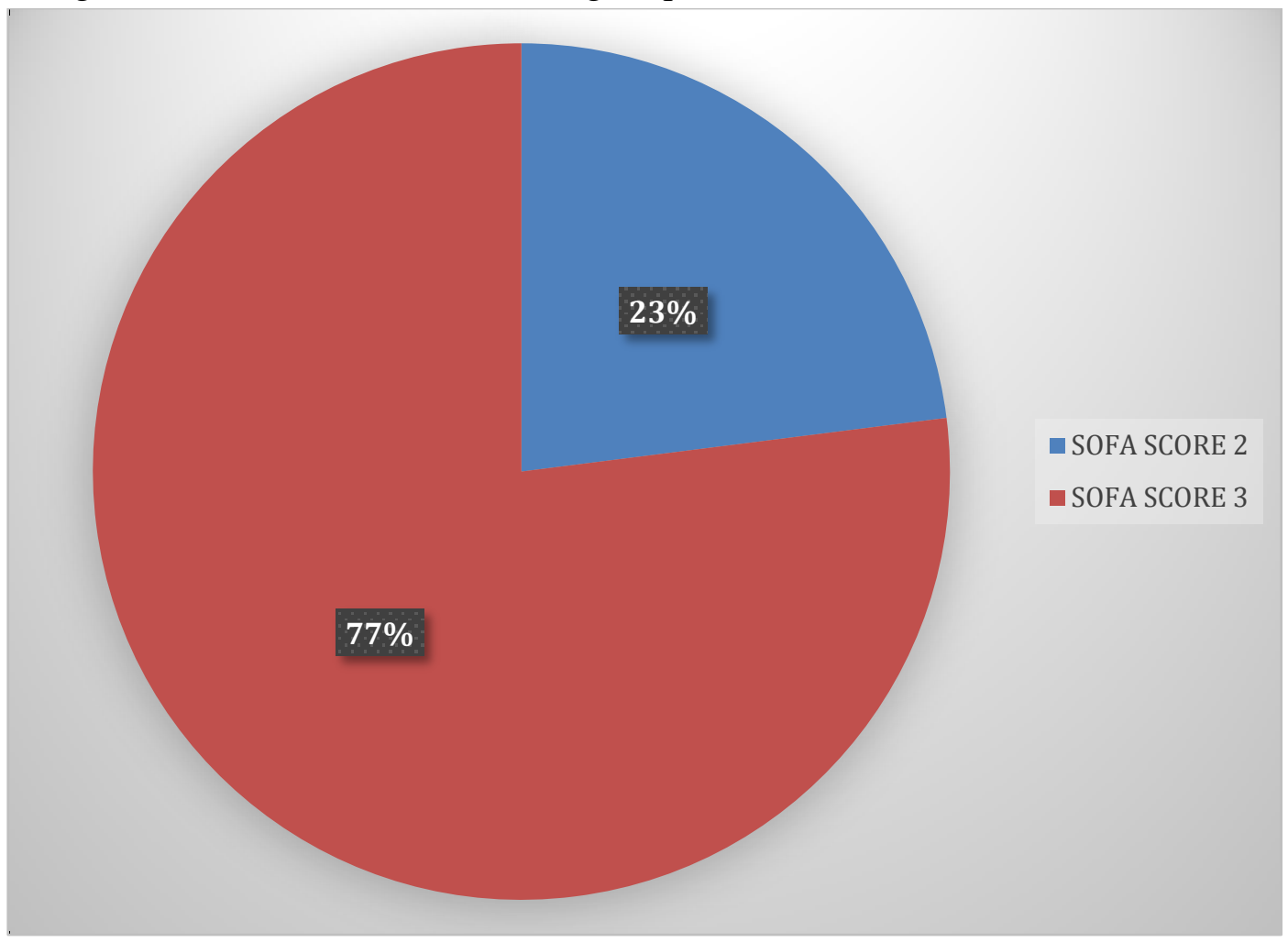

Figure 3: Percentage Distribution of Cases according to AEC

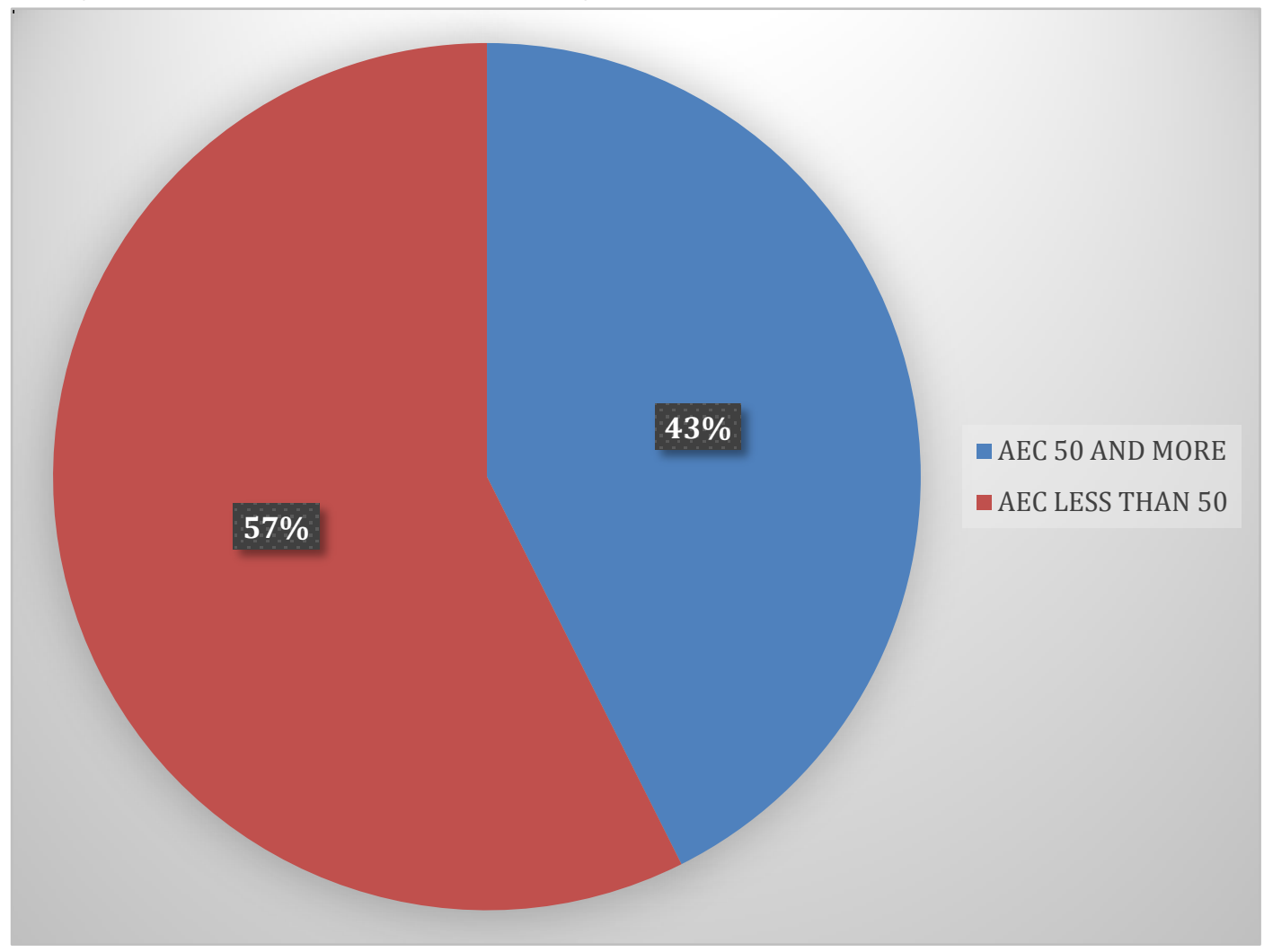


Figure 4: Percentage Distribution of Controls according to AEC

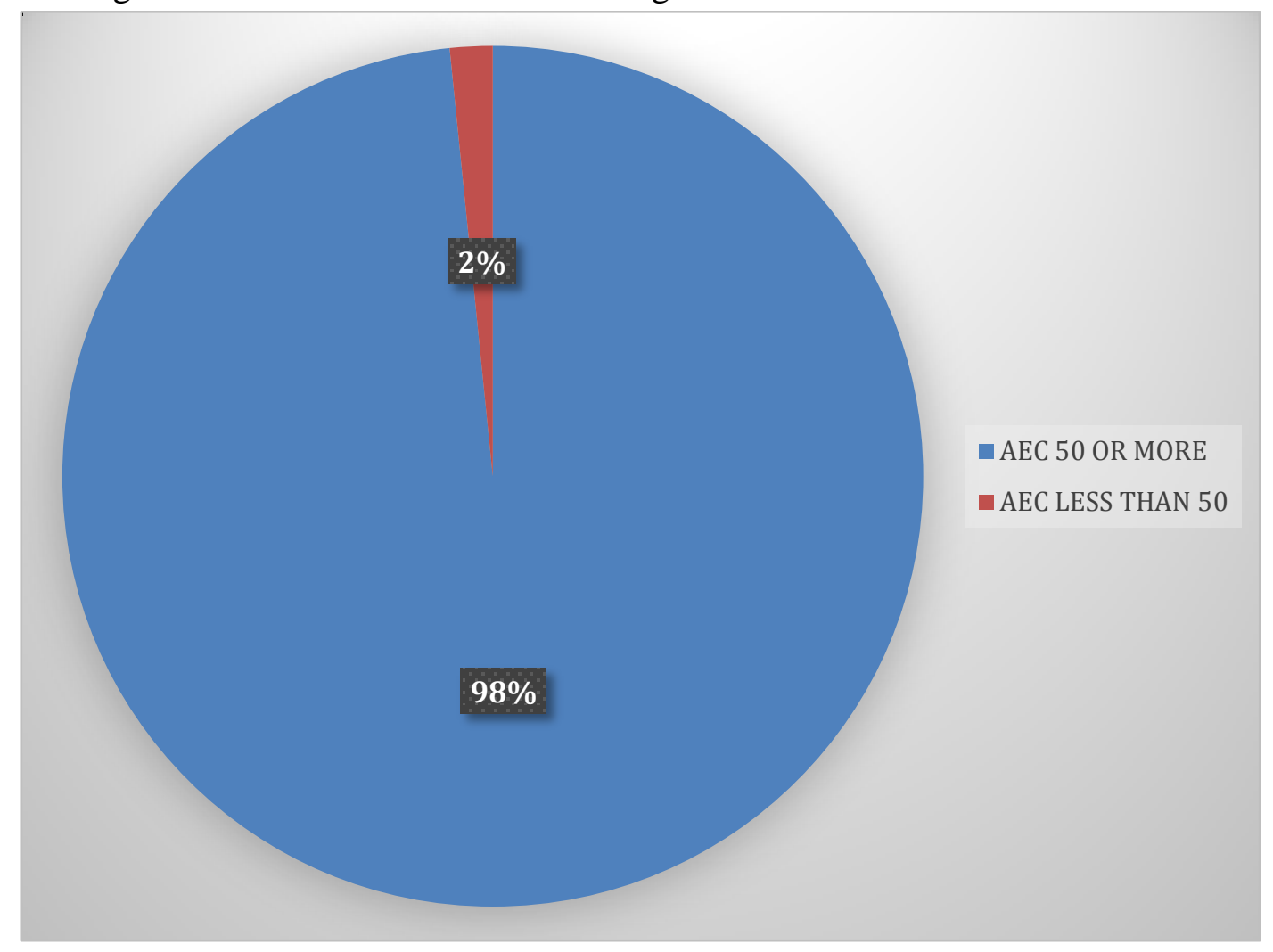

Figure 5: Percentage Distribution of cases According to CRP

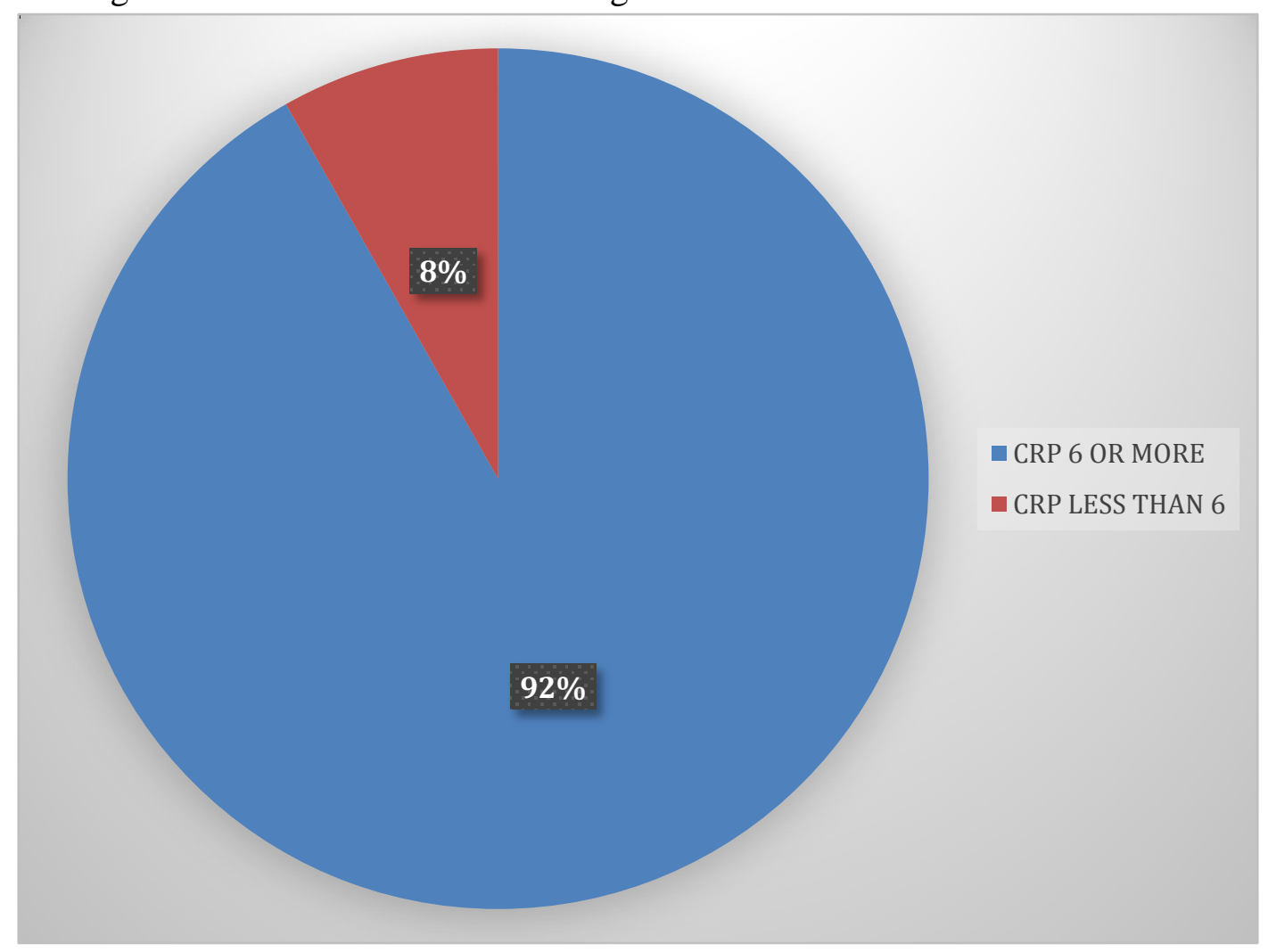




\section{JMSCR Vol||10||Issue||01||Page 213-219||January}

Figure 6: Percentage Distribution of Controls according to CRP

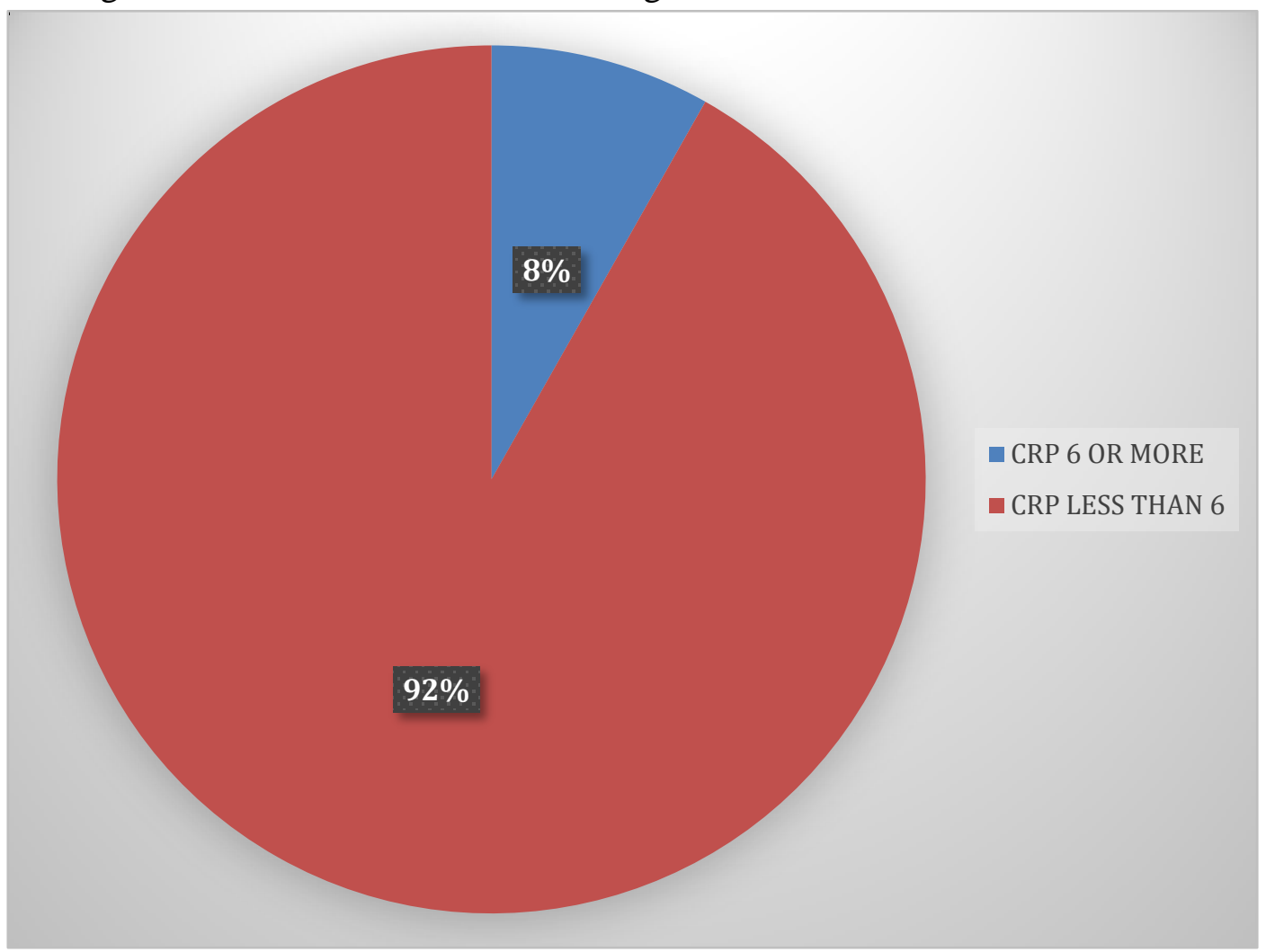

Table 1: Predictive Power of AEC as Diagnostic Marker in Sepsis

\begin{tabular}{|l|c|c|}
\hline \multirow{2}{*}{ Absolute Eosinophil Count } & \multicolumn{2}{|c|}{ Sepsis } \\
\cline { 2 - 3 } & Present & Absent \\
\hline Less than 50 & 35 & 1 \\
\hline More than or equal To 50 & 26 & 60 \\
\hline
\end{tabular}

Sensitivity 57.4\%, Specificity 98.4\%, Positive Predictive Value 97.2\%, Negative Predictive Value 69.8\%, Accuracy 77.9\%

Table 2: Predictive Power of CRP as Diagnostic Marker in Sepsis

\begin{tabular}{|l|c|c|}
\hline \multirow{2}{*}{ CRP } & \multicolumn{2}{|c|}{ Sepsis } \\
\cline { 2 - 3 } & Present & Absent \\
\hline Less than or Equal to 6 & 5 & 56 \\
\hline More Than 6 & 56 & 5 \\
\hline
\end{tabular}

Sensitivity $91.8 \%$, Specificity $91.8 \%$, Positive Predictive Value 91.8\%, Accuracy $91.8 \%$.

Table 3: Comparison of AEC and CRP as a Diagnostic Marker in Sepsis

\begin{tabular}{|l|c|c|c|c|}
\hline & AEC & CRP & $\mathrm{Z}$ & P VALUE \\
\hline Sensitivity & $57.4 \%$ & $91.8 \%$ & 6.176 & $<0.01$ \\
\hline Specificity & $98.4 \%$ & $91.8 \%$ & 2.368 & 0.018 \\
\hline Positive Predictive Value & $97.2 \%$ & $91.8 \%$ & 1.858 & 0.064 \\
\hline Negative Predictive Value & $69.8 \%$ & $91.8 \%$ & 4.368 & $<0.01$ \\
\hline Accuracy & $77.9 \%$ & $91.8 \%$ & 3.034 & 0.002 \\
\hline
\end{tabular}

\section{Best Cut Off For AEC as a Diagnostic Marker} in Sepsis

The best cut off for AEC under the ROC curve is 245. Area under the curve $=0.897$. (95\% CI $0.836-0.959), \mathrm{p}=0.000$ with a sensitivity of $83.6 \%$, specificity of $91.8 \%$ and a Positive Predictive Value of $91.8 \%$.

\section{Best Cut Off For CRP as a Diagnostic Marker in Sepsis}

The best cut off value obtained for CRP as a diagnostic marker in sepsis using an ROC curve is 7. Area under the curve $=0.938(95 \%$ CI 0.892 $0.983), \mathrm{p}=0.000$, with a sensitivity of $91.8 \%$, 
specificity of $91.8 \%$ and a Positive predictive value of $91.8 \%$.

\section{Predictive Power of AEC as a Prognostic Marker in Sepsis}

Kappa $=-0.02, \mathrm{p}=0.854$. Poor agreement, with a sensitivity of $56.9 \%$, specificity of $40 \%$ and accuracy of $54.1 \%$

Predictive Power of CRP as a Prognostic Factor in Sepsis
Kappa $=-0.12, \mathrm{p}=0.301$. Poor agreement, with a sensitivity of $90.2 \%, 0 \%$ specificity and an accuracy of $75.4 \%$

At the best cut off of 35, the sensitivity of AEC as a prognostic marker is $35.3 \%$, specificity is $90 \%$, accuracy of $44.3 \%$. Area under the curve $=0.539(95 \%$ CI $0.391-0.688) \mathrm{p}=0.697$.

At the best cut off of 39 , the sensitivity of CRP is $41.2 \%$, specificity is $60 \%$ and accuracy is $44 \%$. Area under the curve $=0.423(95 \%$ CI 0.248 $0.597), p=0.442$.

Figure 7: Percentage Distribution of Samples according to the end Point

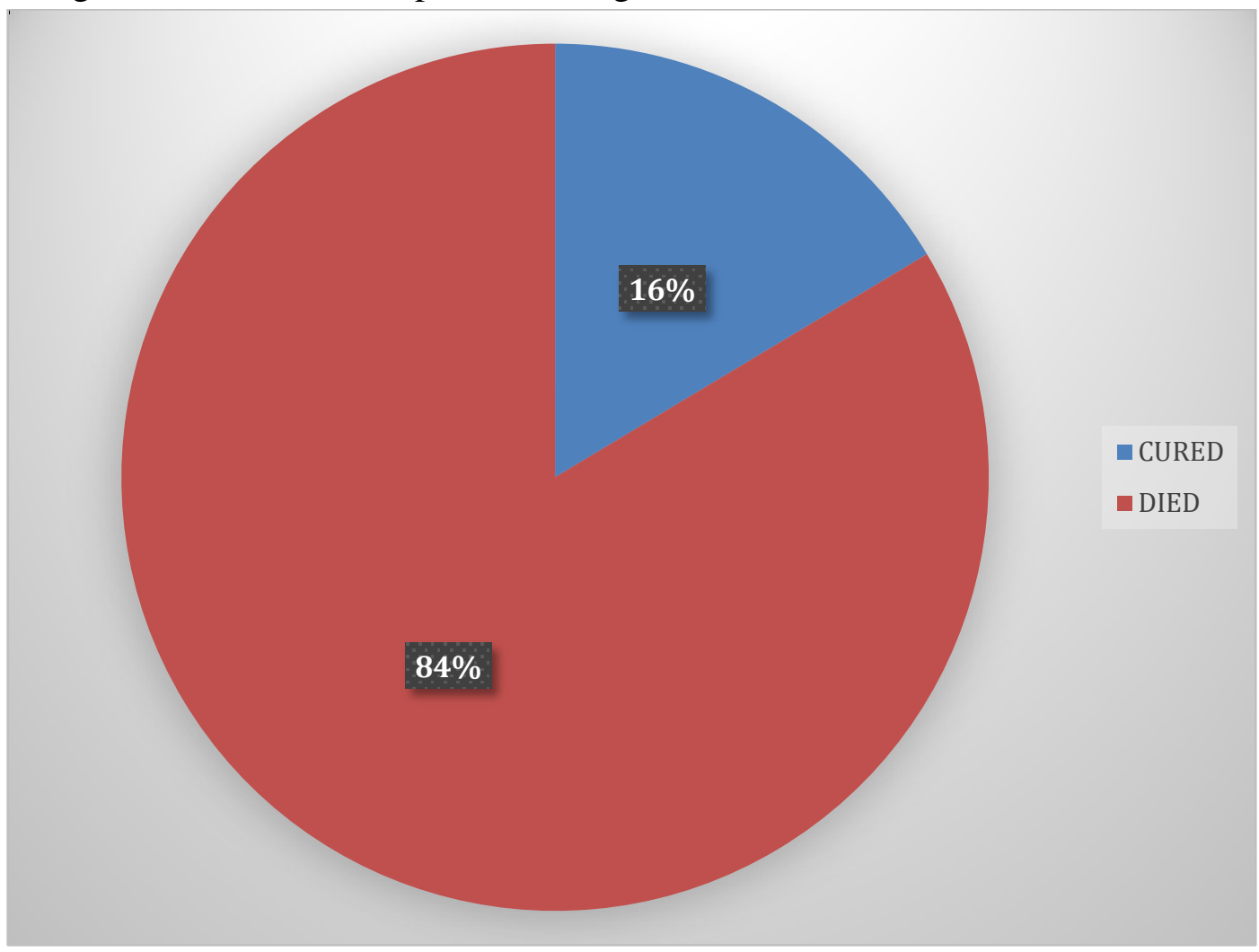

\section{Discussion}

The early diagnosis of sepsis plays an integral role in the morbidity and mortality of sepsis. The clinical parameters that make up sepsis syndrome are not specific and frequently overlap with the presentation of a Systemic Inflammatory Response Syndrome secondary to non infectious causes $^{(2)}$.

Acute infections induce eosinopenia through several mechanisms, like sequestration of eosinophils at the site of inflammation, emergence of mature eosinophils from the bone marrow and suppression of eosinophil production ${ }^{(3)}$. Acute stress also involves eosinopenia, which is mediated by adrenal glucocorticoids and epinephrine.

An early diagnosis of sepsis before microbial cultures are available would certainly facilitate the choice of antibiotic therapy and reduce patient mortality $^{(4)}$.

This is a prospective observational case-control study to compare the accuracy of two biomarkers, Absolute Eosinophil Count and C Reactive Protein as diagnostic and prognostic markers. In 
this study, $\mathrm{C}$ reactive protein of $>6$ and Absolute Eosinophil Count of $<50$ is considered as a biomarker of sepsis.

64 cases were enrolled in the study of which 3 were lost for follow up. Hence the total number of cases were 61. 61 age and sex matched controls were taken from the bystanders of various patients.

Among the cases, $49.2 \%$ were in the age group of $>60$ years. Only $13.1 \%$ cases of sepsis was seen in those less than 40 years of age. $77 \%$ of cases had a qSOFA score of 2 and $23 \%$ had a score of 3 . $57.4 \%$ of the cases had an Absolute Eosinophil count of $<50$. Out of the controls, $98.4 \%$ had an absolute eosinophil count of $>50$. So, the sensitivity, specificity, Positive and Negative Predictive Values of Absolute Eosinophil count in diagnosis of sepsis is $57.4 \%, 98.4 \%, 97.2 \%$ and $69.8 \%$ respectively.

$91.8 \%$ of the cases had C Reactive Protein of $>6$, whereas only $8.2 \%$ of the controls had a C reactive protein of $>6$. Hence, the sensitivity, specificity, Positive Predictive Value and Negative Predictive Value of C Reactive protein is $91.8 \%, 91.8 \%, 91.8 \%$ and $99.8 \%$ respectively.

Therefore, C Reactive Protein has greater sensitivity in diagnosis of sepsis and Absolute Eosinophil Count has a greater specificity.

In a study conducted by Khalid Abidi et al, the sensitivity of AEC is $80 \%$, specificity is $91 \%{ }^{(5)}$. Compared with the study done by Jose GarnachoMontero et al, the sensitivity of $\mathrm{C}$ Reactive Protein is $90.6 \%$, which is comparable to the present study. But our study shows a higher specificity of CRP compared to their study ${ }^{(6)}$.

End point taken in our study was either cured or died. $83.6 \%$ cases of sepsis died in our study. Of the 51 deaths, 46 had CRP more than 6. Among the 10 cases cured, all had a CRP

More than 6. So CRP is a poor predictor of outcome in sepsis. Absolute Eosinophil Count of $<50$ was seen in 29 of the 5eaths, and 6 of the 10 cases who were cured also had an AEC of $<50$.
So according to our study, AEC is also a poor predictor of prognosis in sepsis.

Best cut off of AEC as a prognostic marker is below 35. Area under the curve is 0.539 , p value 0.697 , with sensitivity of $35.4 \%$, specificity of $90 \%$, Positive Predictive Value of $97.4 \%$ and Negative Predictive Value of $21.4 \%$.

Best cut of CRP as a prognostic marker is above 39. Area under the curve is 0.423 , p value 0.697 , with a sensitivity of $41.2 \%$, specificity of $60 \%$, Positive Predictive Value of $84 \%$ and Negative Predictive Value of $84 \%$.

Our study concludes that CRP has greater sensitivity, whereas, AEC has greater specificity in diagnosing sepsis. Both AEC and CRP are poor predictors of outcome. A larger population and a sub group analysis of cause of sepsis may throw further light in this context.

\section{References}

1. Mervyn Singer etal. The third consensus definitions for sepsis and septic shock. JAMA.2016;315(8):801-810.

2. Curtis NS and Wes S. New concepts in sepsis. Curr Opin Crit Care. 2002;8:465470.

3. Bass DA etal. Eosinopenia of acute infection: production of eosinopenia by chemotactic factors of acute inflammation. 1980. J Clin Investig. 65: 1265-1271

4. Luzzani a etal. Comparison of pro calcitonin and $\mathrm{C}$ reactive protein as markers of sepsis. Crit Care Med. 2003.31:1737-1741.

5. Khalid Abidi etal. Eosinopenia is a reliable marker of sepsis on admission to medical intensive care units. Critical Care. 2008. 12:R59:8

6. Jose Garnacho-Montero et al. Prognostic and diagnostic value of eosinopenia, $\mathrm{C}$ reactive protein, pro calcitonin and circulating cell free DNA in critically ill patients admitted with suspicion of sepsis. Critical Care. 2014:18:R116. 